Ich beginue damit, daß ich alle bisher von uns beobachteten Fälle in Form einer Tabelle mitteile, aus der alle belangreichen Daten ersichtlich sind.

Anfänglich beschränkten wir uns darauf, lediglich chininresistente Fälle von brasilianischer Malaria der 606-Behandlung zu unterziehen, später gingen wir dazu über, die Wirksamkeit des Präparates auch bei anderen uns geeignet erscheinenden Malariafällen zu erproben.

Was die Parasitenart anlangt, so sind von den im ganzen behandelten 22 Fällen elf Tropica- und elf Tertianainfektionen.

Wir begannen unsere Untersuchungen über die Wirksamkeit des Präparates 606 bei Malaria bereits im Mai 1910 und haben dem Fortschritt der Injektionstechnik folgend zuerst intramuskulär in die Nates, später subkutan und intravenös injiziert. Unsere letzten Injektionen applizierten wir, dem Rate von Herrn Geheimrat Ehrlich folgend, kombiniert, indem wir einer am ersten Tage verabreichten intravenösen am darauffolgenden Tage eine subkutane Injektion folgen ließen.

Die intramuskuläre Injektion geschah mit alkalischer In-

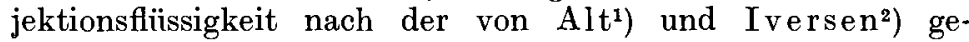
gebenen Vorschrift; die subkutanen Injektionen wurden nach den Vorschriften von WechseImann ${ }^{3}$ ) und Blaschko ${ }^{4}$ ) in neutraler Aufschwemmung vorgenommen. Für die intravenöse Injektion verwandten wir anfänglich die biazide, später die monazide Lösung. An Nebenwirkungen - um das gleich hier vorwegzunehmen - sahen wir anfänglich bei der intramuskulären Injektion alkalischer Lösung starke und sehr lästige Infiltrationen. Bei der subkutanen Injektion neutraler Injektionsaufschwemmung traten zwar auch noch vereinzelte Infiltrationen auf, doch waren diese viel geringer und nur in geringem Grade lästig. Nach den intravenösen Injektionen kam es gewöhnlich zu einem mäßigen Schüttelfrost, der von Erbrechen galliger Massen begleitet oder gefolgt war. Der gleichzeitig eintretende Temperaturanstieg war wohl stets auf die Injektion $\mathrm{zu}$ beziehen, doch war nicht in jedem Falle eine scharfe Ternnung dieser Temperatursteigerung von der durch Malaria bedingten Fieberbewegung möglich.

Die Dosis wurde allmählich gesteigert; anfänglich gaben wir 0,3 und gingen allmählich höher bis auf 0,6 und $0,7 \mathrm{~g}$ in subkutaner bzw. kombinierter Injektion. Die größte intravenös von uns gegebene Menge betrug $0,5 \mathrm{~g}$.

Was nun die Behandlungserge bnisse anlangt, so konnten wir gleich in unseren ersten Fällen cinen sehr deutlichen antiparasitären Einflu $B$ des Mittels feststellen. Diese Wirkung hat sich auch beim Fortgang unserer Untersuchungen bestätigt, doch stellte sich bald ein praktisch und theoretisch sehr interessanter Unterschied heraus zwischen der Wirkung des Mittels auf die Tertianaparasiten im Gegensatz zu der auf die Tropicaparasiten. Tertianaerwies sichals viel weniger resistent gegen 606 als Tropica. Was zunächst das anfängliche Verschwinden der Parasiten aus dem peripherischen Blute anlangt, so trat das bei allen 11 Tertianafällen prompt ein, und zwar $3 \mathrm{mal}$ innerhalb $12,5 \mathrm{mal}$ innerhalb 24 und $3 \mathrm{mal}$ innerhalb 48 Stunden nach der Injektion. Diese prompte Wirkung erstreckte sich sowohl auf die Schizonten wie auf die Gameten. ${ }^{5}$ )

Anders bei der Tropica. Hier verschwanden die Parasiten nur in 5 Fällen unmittelbar nach der Injektion, und zwar $1 \mathrm{mal}$ in $12,1 \mathrm{mal}$ in 24 und $3 \mathrm{mal}$ in 48 Stunden nach der Injektion. Dagegen gelang es in den übrigen 6 Tropicafällen nicht, die Parasiten zum völligen Schwinden zu bringen.

Hamburg. (Leiter: Med.-Rat Prof. Dr. Nocht.)

\section{Das Ehrlich-Hata Mittel 606 bei Malaria.}

Von Dr. H. Werner, Stabsarzt der Schutztruppen und Abteilungsvorsteher der klinischen Abteilung des Instituts.

Nachdem kürzlich die ersten Resultate der Malariabehandlung mit Ehrlichs 606 mitgeteilt worden sind, ${ }^{3}$ ) will ich jetzt über den Fortgang unserer Untersuchungen hinsichtlich der Wirksamkeit des Präparates berichten.

3) Nocht und Werner, Deutsche medizinische Wochenschrift 1910, No. 34, S. 1557
1) Alt, Münchener medizinische Wochenschrift 1910, S. 561.

2) Iversen, Münchener medizinische Wochenschrift 1910, S. 777

3) Wechselmann und Lange, Deutsche medizinische Wochenschrift 1910 , S. 1395 .

4) Blaschko, Berliner klinische Wochenschrift 1910, S. 1611 .

s) Es sei hier bemerkt, da.Bnach unseren Beobachtungen im Seemannskrankenhaus die Tertianagameten sich auch dem Chinin gegenüber viel weniger resistent verhalten als die Tropicagameten; letztere leisten einer energischen Chininbehandlung oft wochenlang Widerstand, wäh rend die Tertianagameten gewöhnlich schon am dritten Tage der Chininbehandlung zu schwinden pflegen. Von den Schizonten da gegen ist zu sagen, daß die der Tropica denen der Tertiana in bezug auf Widerstandsfähigkeit gegen Chinin um ein geringes nachstehen. 
Zwar zeigte sich stets eine deutliche Verminderung, aber auch größere Dosen (0,6 kombiniert) genügten nicht, die Parasiten völlig zum Schwinden zu bringen. Es sei hier ausdrücklich darauf hingewiesen, da $B$ wir bisher die Injektionen nie wiederholt haben. Dieser Umstand muß auch im Auge behalten werden, wenn ich jetzt über das weitere Schicksal der Parasiten berichte.

Auch hier zeigt sich der Unterschied zwischen Tertiana und Tropica. Zunächst die Tertiana: In 8 von den 11 behandelten Fällen haben wir ein Wiederaufreten der Parasiten nicht feststellen können. In 3 Fällen traten dagegen die Parasiten und damit das Fieber nach 4, 7 und 21 Tagen wieder auf. In diesen 3 Fällen betrugen die Dosen $0,3,0,4$ und $0,5 \mathrm{~g}$. Bei den größeren Dosen $(0,6$ und $0,7 \mathrm{~g})$, insbesondere bei kombinierter Verabreichung dieser Dosen (ersten Tag intravenös, zweiten Tag subkutan) haben wir bisher Rezidive bei Tertiana noch nicht beobachtet. Die Beobachtungszeiten sind allerdings, wie aus der Tabelle ersichtlich, zurzeit noch recht kurz, immerhin lang genug, um die Wirksamkeit der Injektion $\mathrm{zu}$ illustrieren.

Viel ungünstiger sind auch hier wieder die Resultate bei der Tropica. Keiner von den fünf Fällen, in denen anfänglich die Parasiten verschwanden, ist rezidivfrei geblieben; vielmehr kehrten die ungeschlechtlichen Parasiten - von den hartnäckigeren Halbmonden sehe ich hier ganz ab - nach durchschnittlich sechs Tagen wieder und wichen erst einer dann eingeleiteten Chinintherapie.

\begin{tabular}{|c|c|c|c|c|c|c|c|c|c|c|c|c|c|c|}
\hline \multirow[b]{2}{*}{$\begin{array}{l}\text { Fall } \\
\mathrm{Nr} .\end{array}$} & \multirow[b]{2}{*}{$\begin{array}{c}\text { Parasitenart. } \\
\text { vor } \\
\text { Injektion }\end{array}$} & \multirow[b]{2}{*}{$\begin{array}{c}\text { Herkunft } \\
\text { der } \\
\text { Malaria }\end{array}$} & \multicolumn{4}{|c|}{ Medikation } & \multicolumn{3}{|c|}{ Schicksal der Parasiten } & \multirow[b]{2}{*}{$\begin{array}{c}\text { Beeinflussung } \\
\text { des } \\
\text { Fiebers }\end{array}$} & \multicolumn{3}{|c|}{ Nebenwirkıngen } & \multirow[b]{2}{*}{ Bemerkungen } \\
\hline & & & $\stackrel{m}{\circ}$ & $\begin{array}{l}\text { Injektions- } \\
\text { technik }\end{array}$ & Reaktion & $\left|\begin{array}{c}\text { Ort } \\
\text { der Injekt. }\end{array}\right|$ & $\begin{array}{l}\text { Anfängliches } \\
\text { Verschwinden }\end{array}$ & $\begin{array}{l}\text { Späteres } \\
\text { Wieder- } \\
\text { auftreten }\end{array}$ & $\begin{array}{c}\text { Be- } \\
\text { obach- } \\
\text { tungs- } \\
\text { daarer } \\
\text { nach d. } \\
\text { lnjekt. }\end{array}$ & & $\begin{array}{l}\text { Schüttel- } \\
\text { frost }\end{array}$ & $\begin{array}{c}\text { Er- } \\
\text { brechen }\end{array}$ & $\begin{array}{l}\text { In- } \\
\text { filtra- } \\
\text { tion }\end{array}$ & \\
\hline 2 & $\mid \begin{array}{l}\text { Tertiana. } \\
\text { Schizonten } \\
\text { Gameten }\end{array}$ & Brasilien & 0,3 & intramuskul. & alkalisch & nates & $\begin{array}{l}\text { Sämtliche Parasiten } \\
\text { (Schizonten 11nd Ga- } \\
\text { meten) verschwunden } \\
\text { innerhalb } 12 \text { stund en } \\
\text { nach der Injektion. } \\
\text { Sämtliche Parasiten ver- } \\
\text { schwinden innerhalb } \\
12 \text { Stunden nach In- } \\
\text { jektion. }\end{array}$ & $\begin{array}{l}\text { Nic h t } \\
\text { wieder auf- } \\
\text { getreten. } \\
\text { Wiederauf- } \\
\text { trelen nach } \\
5 \text { Tagen. }\end{array}$ & 10 Tage. & $\begin{array}{l}\text { Schwinden } \\
\text { innerhalb } \\
12 \text { Stunden } \\
\text { nach der } \\
\text { Injektion. } \\
\text { Schwindeti } \\
\text { innerhalb } \\
12 \text { Stunden } \\
\text { nach der }\end{array}$ & - & - & gering & \\
\hline 3 & $\underset{\substack{\text { Tertiana. } \\
\text { Schizonten } \\
\text { Gameten }}}{+}$ & Brasilien & 0,3 & intramuskul. & alkalisch & nates & \begin{tabular}{|} 
Sämtliche Parasiten ver- \\
schwunden innerhalb \\
12 Stunden nach der \\
Injektion.
\end{tabular} & $\begin{array}{l}\text { Wiederauf- } \\
\text { tre'en nach } \\
21 \text { Tagen. }\end{array}$ & & $\begin{array}{l}\text { Injektion. } \\
\text { Schivinden } \\
\text { intinerhalb } \\
12 \text { Stunden } \\
\text { nach der }\end{array}$ & - & - & stark & \\
\hline 4 & $\begin{array}{l}\text { Tertiana. } \\
\text { Schizonten }\end{array}$ & Brasilien & 0,3 & intramuskul. & alkalisch & nates & $\begin{array}{c}\text { Sämtliche Parasiten ver. } \\
\text { schwunden innerhalb } \\
48 \text { Stunden nach der } \\
\text { Iniektion }\end{array}$ & $\mid \begin{array}{c}\text { Nicht } \\
\text { wieder auf- } \\
\text { getreten. }\end{array}$ & 4 Tage. & $\begin{array}{c}\text { Injektion. } \\
\text { Schwinden } \\
\text { innerhatb } \\
48 \text { Stunden. }\end{array}$ & $\begin{array}{l}+4 \text { Std. } \\
\text { nach } \\
\text { Injekt. }\end{array}$ & - & stark & $\begin{array}{l}4 \text { und } 5 \text { derselbe } \\
\text { Patient } \\
\text { (Mischinfektion) }\end{array}$ \\
\hline 5 & $\begin{array}{l}\text { Tropica. } \\
\text { Schizonten }\end{array}$ & Brasilien & 0,3 & intramuskul. & alkalisch & nates & $\begin{array}{l}\text { Injektion. } \\
\text { Sämtliche Parasiten ver- } \\
\text { schwulden innerhalb } \\
48 \text { Stunden nach I.I- } \\
\text { jektion }\end{array}$ & $\begin{array}{c}\text { Wiederauf- } \\
\text { treten nach } \\
3 \text { Tagen. }\end{array}$ & & $\begin{array}{l}\text { Schwinden } \\
\text { innerhatb } \\
48 \text { Stunden. }\end{array}$ & $\begin{array}{c}+4 \text { Std. } \\
\text { nach } \\
\text { Injekt. }\end{array}$ & - & stark & $\begin{array}{l}4 \text { und } 5 \text { derselbe } \\
\text { Patient } \\
\text { (Misihinfektion) }\end{array}$ \\
\hline 6 & $\begin{array}{l}\text { Tropica. } \\
\text { Schizonten } \\
\text { Gameten }\end{array}$ & Brasilien & 0,3 & intramuskul. & alkalisch & nates & $\mathrm{Nicht}$ verschwunden. & & & $\begin{array}{l}\text { Nicht } \\
\text { ver- } \\
\text { srhwunden. }\end{array}$ & - & - & stark & \\
\hline 8 & $\begin{array}{l}\text { Tertiana. } \\
\text { Schizonten } \\
\text { Gameten }\end{array}$ & Brasilien & 0,6 & subkutan & neutral & Rücken & $\begin{array}{l}\text { Versch winden sämt- } \\
\text { licher Parasiten inner- } \\
\text { halb } 48 \text { itunden nach } \\
\text { Injektion. }\end{array}$ & $\begin{array}{c}\text { Nic h t } \\
\text { wieder auf- } \\
\text { getreten. }\end{array}$ & 8 Tage. & $\begin{array}{l}\text { Nicht ver- } \\
\text { sctiwunden } \\
\text { (infoge } \\
\text { gleichzertig } \\
\text { besteheslder } \\
\text { unbeeinflus- } \\
\text { ler Tr.ppica). } \\
\text { Nicht } \\
\text { ver- }\end{array}$ & - & - & stark & $\begin{array}{l}7 \text { und } 8 \text { be- } \\
\text { treften densel- } \\
\text { ben Patienten } \\
\text { (Misch- } \\
\text { infektion) }\end{array}$ \\
\hline 9 & $\underset{\substack{\text { Tertiana. } \\
\text { Schizoilten } \\
\text { Gameten }}}{+}$ & Brasilien & 0,4 & intravenös & biazid. & Armvelle & $\begin{array}{l}\text { Säıntliche Parasiten ver- } \\
\text { sc'vwunden int»rhalb } \\
24 \text { St nden nach der } \\
\text { Injektion. }\end{array}$ & $\begin{array}{l}\text { Wiederauf- } \\
\text { treten nach } \\
4 \text { Tagen. }\end{array}$ & & $\begin{array}{l}\text { schwunden. } \\
\text { Weder vor } \\
\text { noch nach der } \\
\text { Injektion be- }\end{array}$ & $\begin{array}{c}+3 / 4 \text { Std. } \\
\text { mach } \\
\text { Injekt. }\end{array}$ & $\begin{array}{l}+3 / 4 \text { Stun- } \\
\text { den nach } \\
\text { Injektion }\end{array}$ & - & \\
\hline 10 & $\begin{array}{l}\text { Tertiana. } \\
\text { Schizonten } \\
\text { Gameten }\end{array}$ & Brasilien & 0,5 & intravenös & monaz. & Armvene & $\begin{array}{l}\text { Sämtliche Parasiten ver- } \\
\text { sc wunden !innerhalb } \\
12 \text { Stunden nach In- } \\
\text { jektion. }\end{array}$ & $\begin{array}{c}\text { Wiederauf- } \\
\text { treten nach } \\
10 \text { Tagen. }\end{array}$ & & $\begin{array}{l}\text { Sand Fi ber. } \\
\text { Schwinden } \\
\text { innernalb } \\
12 \text { Stundetl. }\end{array}$ & $\begin{array}{c}+1 / 3 \text { std. } \\
\text { Injekt. }\end{array}$ & $\mid \begin{array}{c}+1 / 2 \\
\text { Stunde } \\
\text { nacti ln- } \\
\text { jektion }\end{array}$ & & 10 und 11 be- \\
\hline 11 & $\begin{array}{l}\text { Tropica. } \\
\text { Gameten }\end{array}$ & Brasilien & 0,5 & intravenös & monaz. & Armvene & $\begin{array}{l}\text { Tropicagameten nicht } \\
\text { verschwunden. }\end{array}$ & $\begin{array}{l}\text { Wiederallf- } \\
\text { treten von } \\
\text { Schiz } z^{\prime} \text { nten } \\
\text { nach7 } 7 \text { ygen. }\end{array}$ & & $\begin{array}{l}\text { Schwinden } \\
\text { in nerhalb } \\
12 \text { Stunden. }\end{array}$ & $\begin{array}{c}+1 / 2 \text { Std. } \\
\text { nach } \\
\text { Injekt. }\end{array}$ & $\begin{array}{c}+1 / 2 \\
\text { Stunde } \\
\text { nach In- } \\
\text { jektion }\end{array}$ & & $\begin{array}{c}\text { ben Patienten } \\
\text { (Misch- } \\
\text { infektion) }\end{array}$ \\
\hline 12 & $\mid \begin{array}{l}\text { Tropica. } \\
\text { Schizonten } \\
\text { Gameten }\end{array}$ & $\begin{array}{l}\text { West- } \\
\text { afrika }\end{array}$ & 0,5 & intravenös & monaz. & Armvene & $\begin{array}{l}\text { Sämtliche Parasiten ver- } \\
\text { schwunden nnerhalb } \\
24 \text { S unden nach der } \\
\text { Injektion. }\end{array}$ & $\begin{array}{l}\text { Wiederauf- } \\
\text { treten nach } \\
6 \text { Tagen. }\end{array}$ & & $\begin{array}{l}\text { Schwinden } \\
\text { innerhalb } \\
48 \text { Stunden. }\end{array}$ & $\begin{array}{c}+1 / 2 \text { Std. } \\
\text { nach } \\
\text { Injekt. }\end{array}$ & $\begin{array}{c}+y_{2} \\
\text { Stunde } \\
\text { nach In- } \\
\text { jektion }\end{array}$ & - & \\
\hline 13 & $\underset{\substack{\text { Tertiana. } \\
\text { Schizonten }}}{\text { Gameten }}$ & Brasilien & 0,5 & intravenös & monaz. & Armvene & $\begin{array}{l}\text { Sămtliche Parasiten ver- } \\
\text { schwunden inuerhalb } \\
24 \text { Stunden 11ach der } \\
\text { Iniektion }\end{array}$ & $\begin{array}{c}\text { Nicht } \\
\text { wieder auf- } \\
\text { getreten. }\end{array}$ & 17 Tage & $\begin{array}{l}\text { Schwinden } \\
\text { innerhalb } \\
12 \text { stunden. }\end{array}$ & $\begin{array}{c}+1 \text { Std. } \\
\text { nach } \\
\text { Injekt. }\end{array}$ & $\begin{array}{l}+3 \text { Stun- } \\
\text { den nach } \\
\text { Injektion }\end{array}$ & - & \\
\hline 14 & $\mid \begin{array}{l}\text { Tertiana. } \\
\text { Schizonten } \\
\text { Gameten }\end{array}$ & $\begin{array}{c}\text { Schwarz } \\
\text { Meer }\end{array}$ & 0,5 & intravenös & monaz. & A'mvene & $\begin{array}{l}\text { Säm.licne Parasiten } \\
\text { innerhalb } 12 \text { Stunden } \\
\text { nich der Injektion } \\
\text { verschwunden }\end{array}$ & $\mid \begin{array}{c}\mathrm{Nic} \text { c h t } \\
\text { wieder } \\
\text { getreten. }\end{array}$ & 9 Tage. & $\begin{array}{l}\text { Schwinden } \\
\text { innerhalb } \\
1 \text { S. Stu.den. }\end{array}$ & $\begin{array}{c}+4 \text { Std. } \\
\text { nach } \\
\text { Injekt. }\end{array}$ & $\begin{array}{l}+6 \text { Stun- } \\
\text { den nach } \\
\text { Injektion }\end{array}$ & - & \\
\hline 15 & $\begin{array}{l}\text { Tertiana. } \\
\text { Schizonten }\end{array}$ & $\begin{array}{c}\text { Mittel- } \\
\text { amerika }\end{array}$ & 0,6 & $\begin{array}{l}\text { kombiniert } \\
\text { intravenoss } \\
+ \text { subkutan }\end{array}$ & $\begin{array}{l}\text { monaz. } \\
\text { bezw. } \\
\text { neutral }\end{array}$ & $\begin{array}{l}\text { Armvene } \\
\text { bezw. } \\
\text { Rücken }\end{array}$ & $\begin{array}{l}\text { verschwunden. } \\
\text { SämtlicheParasitenver- } \\
\text { s h hwun en innerhalb } \\
\text { 24 Stunden nach In- } \\
\text { jektion }\end{array}$ & $\mid \begin{array}{c}\mathrm{Nicht} \\
\text { wieder auf- } \\
\text { getreten. }\end{array}$ & 12 Tage & $\begin{array}{l}\text { Schwinden } \\
\text { innerhalb } \\
12 \text { Stunden. }\end{array}$ & $\mid \begin{array}{c}+15 \mathrm{Mi}- \\
\text { n11ien } \\
\text { nach }\end{array}$ & $\begin{array}{l}+15 \mathrm{Mi}- \\
\text { nuten } \\
\text { nach In- } \\
\text { iektion }\end{array}$ & - & \\
\hline 16 & $\begin{array}{l}\text { Tertiana. } \\
\text { Schizonten } \\
\text { Tropica. } \\
\text { Schizonten } \\
\text { Gameten }\end{array}$ & Brasiliell & 0,7 & $\begin{array}{l}\text { kombiniert } \\
\text { intravenös } \\
\text { + subkutan } \\
\text { kombiniert } \\
\text { intravenös } \\
\text { + subkutan }\end{array}$ & $\begin{array}{l}\text { monaz. } \\
\text { bezw. } \\
\text { neutral } \\
\text { monaz. } \\
\text { b } \simeq z w . \\
\text { neutral }\end{array}$ & $\begin{array}{l}\text { Armvene } \\
\text { bezw. } \\
\text { Rücken } \\
\text { Armvene } \\
\text { bezw. } \\
\text { Rücken }\end{array}$ & $\begin{array}{l}\text { Sämtliche Parasiten ver- } \\
\text { schwunden innerhalb } \\
24 \text { Stunden. } \\
\mathrm{N} \text { ich t verschwunden. }\end{array}$ & $\mid \begin{array}{c}\text { Niclit } \\
\text { wieder auf- } \\
\text { getreten }\end{array}$ & 7 Tage. & $\begin{array}{c}\text { Schwinden } \\
\text { innerhaib } \\
24 \text { Sunden. } \\
\text { Ver- } \\
\text { srhwunden } \\
\text { innerhalb }\end{array}$ & Injekt. & $+\underset{\text { nach }}{15 \mathrm{Min}}$ & stark & \\
\hline 18 & $\begin{array}{l}\text { Tertiana. } \\
\text { Schizonten } \\
\text { Gameten }\end{array}+$ & Mexiko & 0,6 & $\begin{array}{l}\text { kombiniert } \\
\text { intravenōs } \\
+ \text { subkutan }\end{array}$ & $\begin{array}{l}\text { monaz. } \\
\text { bezw. } \\
\text { neutral }\end{array}$ & $\begin{array}{l}\text { Armvene } \\
\text { bezw. } \\
\text { Rücken }\end{array}$ & $\begin{array}{l}\text { Sämtliche Parasiten ver- } \\
\text { sch wunden innerhalb } \\
48 \text { Stunden nach } I n- \\
\text { jektion. }\end{array}$ & $\begin{array}{l}\text { Nich t } \\
\text { wieder auf- } \\
\text { getreten. }\end{array}$ & 12 Tage & $\begin{array}{c}5 \text { Tagen. } \\
\text { Ver- } \\
\text { schwunden } \\
\text { innerhalb } \\
24 \text { Stunden. }\end{array}$ & - & - & mäBig & \\
\hline 19 & $\begin{array}{l}\text { Tropica. } \\
\text { Schizonten }\end{array}$ & $\begin{array}{l}\text { West- } \\
\text { afrika }\end{array}$ & 0,6 & $\begin{array}{l}\text { kombiniert } \\
\text { intravenös } \\
+ \text { subkutan }\end{array}$ & $\begin{array}{l}\text { monaz. } \\
\text { bezw. } \\
\text { neutral }\end{array}$ & $\begin{array}{l}\text { Armvene } \\
\text { bezw. } \\
\text { Rücken }\end{array}$ & $\mathrm{Nicht}$ varschwunden. & & & $\begin{array}{l}\text { Nicht } \\
\text { ver- } \\
\text { schwunden. }\end{array}$ & $\begin{array}{c}\text { Leichiter } \\
\text { Schwin- } \\
\text { del } 1 / 2 \text { Std. } \\
r \text { ach }\end{array}$ & 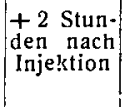 & stark & \\
\hline 20 & $\begin{array}{l}\text { Tropica. } \\
\text { Schizonten }\end{array}$ & Indien & $0,5^{\prime}$ & subkutan & neutral & Rücken & Nicht verschwunden. & & & $\begin{array}{c}\text { Nicht } \\
\text { ver. } \\
\text { schwunden }\end{array}$ & Injekt. & Durchfall & - & \\
\hline 21 & $\begin{array}{l}\text { Tropica. } \\
\text { Schizonten }\end{array}$ & Indien & 0,6 & subkutan & neutral & Rücken & $\begin{array}{l}\text { Sämtliche Parasiten } \\
\text { innerhalb } 48 \text { Stunden } \\
\text { verscnwunden. }\end{array}$ & $\begin{array}{c}\text { Wiederauf- } \\
\text { treten nach } \\
4 \text { Tagen. }\end{array}$ & & $\begin{array}{c}\text { Ve schwinden } \\
\text { innerhalb } \\
24 \text { Stunden. }\end{array}$ & - & - & - & \\
\hline 22 & $\begin{array}{l}\text { Tropica. } \\
\text { Schizonten }\end{array}$ & $\begin{array}{l}\text { West- } \\
\text { afrika }\end{array}$ & 0,6 & $\begin{array}{c}\text { kombiniert } \\
\text { intravenös } \\
+ \text { subkutan }\end{array}$ & $\begin{array}{l}\text { monaz. } \\
\text { bezw. } \\
\text { neutral }\end{array}$ & $\left|\begin{array}{c}\text { Armvene } \\
\text { bezw. } \\
\text { Rücken }\end{array}\right|$ & $\begin{array}{l}\text { Sämtliche Parasiten } \\
\text { i:ınerhalb 24 Stunden } \\
\text { verschwunden. }\end{array}$ & \begin{tabular}{|c|} 
Wiederauf- \\
treten nach \\
6 Tagen.
\end{tabular} & & $\mid \begin{array}{c}\text { Verschwinden } \\
\text { innerhalb } \\
24 \text { Stunden. }\end{array}$ & $\left|\begin{array}{c}\text { nach } \\
\text { Injekt. }\end{array}\right|$ & $\mid \begin{array}{l}+5 \text { Stun- } \\
\text { den nach } \\
\text { Injektion }\end{array}$ & - & \\
\hline
\end{tabular}


Nach alledem kann man sagen, daß das Präparat Ehrlich Hata 606 ein sehr wirksames antiparasitäres Mittel bei Malaria ist, und zwar kommt seine Anwendung in erster Linie in Betracht in allen den Fällen, in denen Chinin versagt, also bei Fällen von Chininresistenz der Malaria und bei Chininintoleranz des Kranken. Ob auch für Schwarzwasserfieberdisposition die 606-Behandlung angezeigt ist, muß weitere Beobachtung lehren. Daß die antiparasitäre Wirkung bei der Tropica teilweise versagt hat, will meines Erachtens zunächst noch nicht viel besagen, da wir ja nur einmalige Injektion angewandt haben und bekanntlich eine einmalige Chiningabe auch nicht imstande ist, die Malaria zu beseitigen. Eine Ueber legenheit von 606 gegenüber dem Chinin scheint für die meisten Fälle von Malaria - wenn man von den beiden eben genannten Kategorien von Malariafällen, in denen Chinin nicht erfolgreich, bzw. kontraindiziert ist, absieht - nicht zu bestehen; immerhin scheint es mir eine sehr willkommene Bereicherung unseres Rüstzeuges im Kampfe gegen die Malaria darzustellen.

Von hohem biologischen Interesse scheint mir der Unterschied der Wirkung auf Tertiana gegenüber der auf die Tropica zu sein; ist das doch ein neuer Hinweis darauf, daB wir es bei Tertiana und Tropica mit nicht nur morphologisch, sondern auch biologisch in hohem Grade voneinander abweichenden Protozoënformen zu tun haben, die im Sinne der Unitätslehre unter eine Art zu rubrizieren kaum noch möglich sein dürfte.

Natürlich haben wir bei unserem therapeutischen Vorgehen auch an die Kombinierung der 606-Medikation mit anderen antimalarischen Mitteln (Chinin und Methylenblau) gedacht, doch haben wir fürs erste von der Ausführung dieser Absicht Abstand genommen, um zunächst die reine Wirkung von 606 feststellen zu können.

Zusammenfassung. 1. 606 ist ein sehr wirksames Antimalaricum.

2. Es wirkt in stärkerem Maße gegen Tertiana als gegen Tropica.

3. Eine einmalige Dosis von 0,6 (kombiniert intravenös und subkutan) genügte in unseren Fällen, die Tertianaparasiten zum Schwinden aus dem peripherischen Blute zu bringen und das Fieber zu beseitigen. Diese Wirkung wurde in durchschnittlich 24 Stunden erzielt. Ob diese Wirkung von langer Dauer ist, kann jetzt noch nicht übersehen werden, jedenfalls haben wir bei mehrwöchiger Beobachtung noch keine Rezidive gesehen.

4. Bei Tropica genügte in etwa der Hälfte der Fälle die Dosis von $\mathbf{0 , 6}$, die Parasiten aus dem peripherischen Blute zeitweilig zu vertreiben, doch traten gewöhnlich schon nach wenigen Tagen die Parasiten und mit ihnen das Fieber wieder auf.

5. Die Anwendung von 606 empfiehlt sich bei Malaria in solchen Fällen, in denen Chinin versagt (Chininresistenz der Malaria) und bei Chininintoleranz. 